Provided for non-commercial research and education use. Not for reproduction, distribution or commercial use.

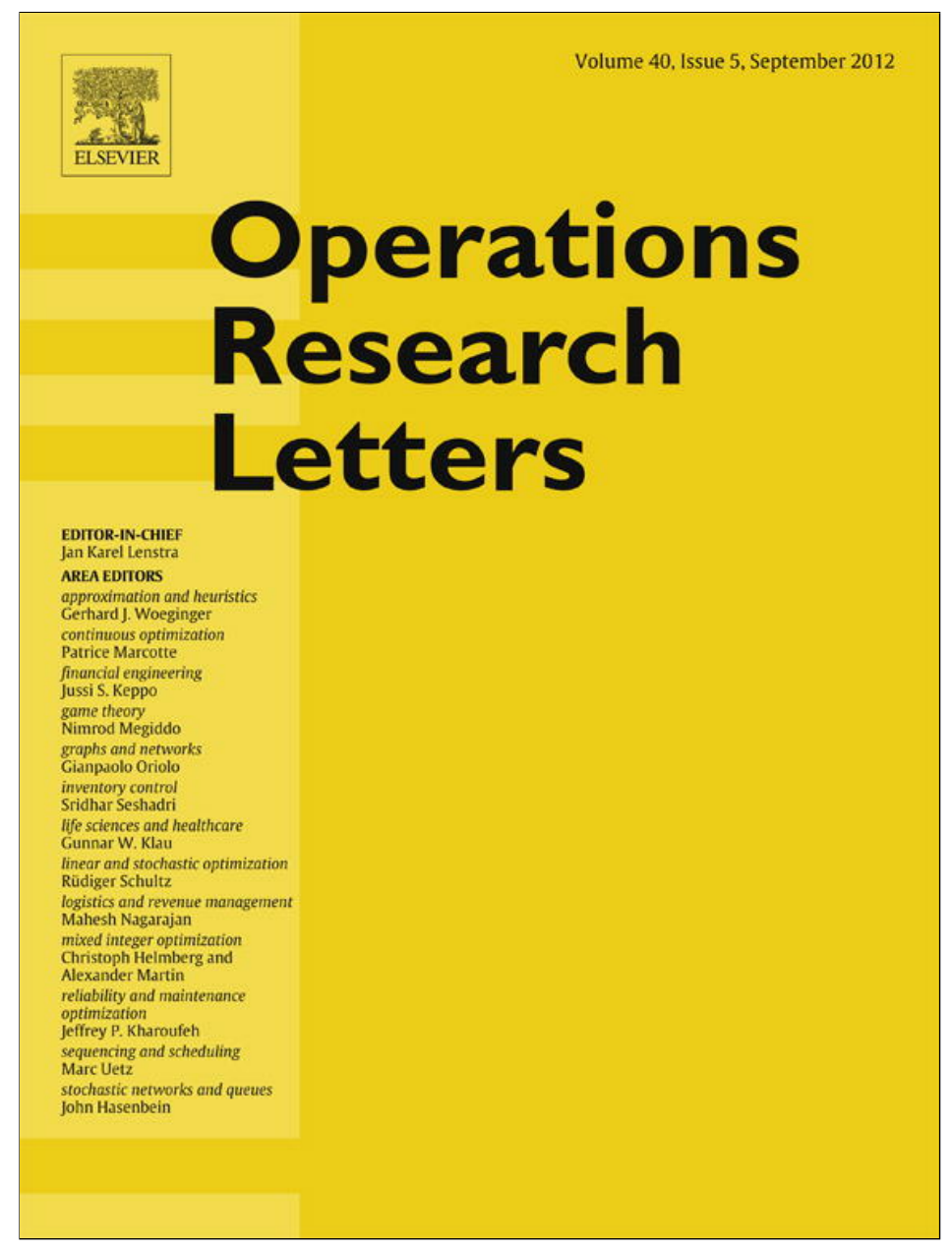

This article appeared in a journal published by Elsevier. The attached copy is furnished to the author for internal non-commercial research and education use, including for instruction at the authors institution and sharing with colleagues.

Other uses, including reproduction and distribution, or selling or licensing copies, or posting to personal, institutional or third party websites are prohibited.

In most cases authors are permitted to post their version of the article (e.g. in Word or Tex form) to their personal website or institutional repository. Authors requiring further information regarding Elsevier's archiving and manuscript policies are encouraged to visit:

http://www.elsevier.com/copyright 


\title{
SDDP for multistage stochastic linear programs based on spectral risk measures
}

\author{
Vincent Guigues $^{\mathrm{a}, \mathrm{b}}$, Werner Römisch ${ }^{\mathrm{c}, *}$ \\ a IMPA, Instituto de Matemática Pura e Aplicada, 110 Estrada Dona Castorina, Jardim Botanico, Rio de Janeiro, Brazil \\ ${ }^{\mathrm{b}}$ UFRJ, Escola Politécnica, Departamento de Engenharia Industrial, Ilha do Fundão, CT, Bloco F, Rio de Janeiro, Brazil \\ ${ }^{\mathrm{c}}$ Humboldt-University Berlin, Institute of Mathematics, 10099 Berlin, Germany
}

\section{A R T I C L E I N F O}

\section{Article history:}

Received 8 February 2012

Received in revised form

29 March 2012

Accepted 10 April 2012

Available online 15 May 2012

\section{Keywords:}

Spectral risk measure

Stochastic programming

Risk-averse optimization

Decomposition algorithms

Monte Carlo sampling

\begin{abstract}
A B S T R A C T
We consider risk-averse formulations of multistage stochastic linear programs. For these formulations, based on convex combinations of spectral risk measures, risk-averse dynamic programming equations can be written. As a result, the Stochastic Dual Dynamic Programming (SDDP) algorithm can be used to obtain approximations of the corresponding risk-averse recourse functions. This allows us to define a risk-averse nonanticipative feasible policy for the stochastic linear program. Formulas for the cuts that approximate the recourse functions are given. In particular, we show that some cut coefficients have analytic formulas.
\end{abstract}

(C) 2012 Elsevier B.V. All rights reserved.

\section{Introduction}

Multistage stochastic programs play a central role when developing optimization models under stochastic uncertainty in engineering, transportation, finance, and energy. Furthermore, since measuring, bounding, or minimizing the risk of decisions becomes more and more important in applications, risk-averse formulations of such optimization models are needed and have to be solved. Several risk-averse model variants allow for a reformulation as a classical multistage model, as in $[6,8]$ and the present paper. From a mathematical point of view, multistage stochastic optimization methods represent infinite-dimensional models in spaces of random vectors satisfying certain moment conditions and contain high-dimensional integrals. Hence, their numerical solution is a challenging task. Each solution approach consists at least of two ingredients: (i) numerical integration methods for computing the expectation functionals and (ii) algorithms for solving the resulting finite-dimensional optimization models.

The favorite approach for (i) is to generate possible scenarios (i.e., realizations) of the random vector involved and to use them as 'grid points' for the numerical integration. Scenario generation can be done by Monte Carlo, quasi-Monte Carlo, or optimal quantization methods (see $[5,18]$ for overviews and [3, Part III] for

\footnotetext{
* Corresponding author. Tel.: +49 3020932561.

E-mail addresses: vguigues@impa.br (V. Guigues),romisch@math.hu-berlin.de (W. Römisch).
}

further information). Scenarios for multistage stochastic programs have to be tree structured to model the increasing chain of $\sigma$ fields. Existing stability and convergence results such as those in $[11,10,12,21]$ provide approaches and conditions implying the convergence of such schemes, in particular, for the deterministic first-stage solutions. Hence, they justify rolling horizon approaches based on repeated solving of multistage models; see [9], for instance.

The algorithms employed for (ii) depend on structural properties of the basic optimization model and on the inherent structure induced by the scenario tree approximation (see the survey [19] on decomposition methods).

Some algorithmic approaches incorporate the scenario generation method (i) as an algorithmic step of the solution method. Such approaches are, for example, stochastic decomposition methods for multistage models (see [20]), approximate dynamic programming (see [17]), and Stochastic Dual Dynamic Programming (SDDP), initiated in [13], revisited in [16,22], and also studied in the present paper.

We consider risk-averse formulations of multistage stochastic linear programs of the form

$$
\begin{aligned}
& \inf _{x_{1}, \ldots, x_{T}} d_{1}^{\top} x_{1}+\theta_{1} \mathbb{E}\left[\sum_{t=2}^{T} d_{t}^{\top} x_{t}\right]+\sum_{t=2}^{T} \theta_{t} \rho_{\phi}\left(-\sum_{k=2}^{t} d_{k}^{\top} x_{k}\right) \\
& C_{t} x_{t}=\xi_{t}-D_{t} x_{t-1}, \\
& x_{t} \geq 0, \quad x_{t} \text { is } \mathcal{F}_{t} \text {-measurable, } t=1, \ldots, T,
\end{aligned}
$$


where $x_{0}$ is given, parameters $d_{t}, C_{t}, D_{t}$ are deterministic, $\left(\xi_{t}\right)_{t=1}^{T}$ is a stochastic process, $\mathcal{F}_{t}$ is the sigma-algebra $\mathcal{F}_{t}:=\sigma\left(\xi_{j}, j \leq t\right)$, $\left(\theta_{t}\right)_{t=1}^{T}$ are nonnegative weights summing to 1 , and $\rho_{\phi}$ is a spectral risk measure [1] or distortion risk measure [14,15] depending on a risk spectrum $\phi \in L_{1}([0,1])$. In the above formulation, we have assumed that the (one-period) spectral risk measure takes as argument a random income and that the trajectory of the process is known until the first stage. We assume relatively complete recourse for (1), which means that, for any feasible sequence of decisions $\left(x_{1}, \ldots, x_{t}\right)$ to any $t$-stage scenario $\left(\xi_{1}, \xi_{2}, \ldots, \xi_{t}\right)$, there exists a sequence of feasible decisions $\left(x_{t+1}, \ldots, x_{T}\right)$ with probability 1 . A non-risk-averse model amounts to taking $\theta_{1}=1$ and $\theta_{t}=0$ for $t=2, \ldots, T$. A more general risk-averse formulation for multistage stochastic programs is considered in [8]. For these models, dynamic programming (DP) equations are written in [8] and an SDDP algorithm is detailed to obtain approximations of the corresponding recourse functions in the form of cuts. The main contribution of this paper is to provide analytic formulas for some cut coefficients, independent of the sampled scenarios, that can be useful for implementation. We also specialize the SDDP algorithm and especially the computation of the cuts for the particular riskaverse model (1).

We start by setting down some notation.

- e will denote a column vector of all 1s;

- for $x, y \in \mathbb{R}^{n}$, the vector $x \circ y \in \mathbb{R}^{n}$ is defined by $(x \circ y)(i)=$ $x(i) y(i), i=1, \ldots, n$;

- for $x \in \mathbb{R}^{n}$, the vector $x^{+} \in \mathbb{R}^{n}$ is defined by $x^{+}(i)=$ $\max (x(i), 0), i=1, \ldots, n$;

- the available history of the process at stage $t$ is denoted by $\xi_{[t]}:=\left(\xi_{j}, j \leq t\right)$

- for vectors $x_{1}, \ldots, x_{n}$, the notation $x_{n_{1}: n_{2}}$ stands for the concatenation $\left(x_{n_{1}}, x_{n_{1}+1}, \ldots, x_{n_{2}}\right)$ for $1 \leq n_{1} \leq n_{2} \leq n$;

- $\delta_{i j}$ is the Kronecker delta defined for $i, j$ integers by $\delta_{i j}=1$ if $i=j$ and 0 otherwise.

\section{Risk-averse dynamic programming}

Let $F_{Z}(x)=\mathbb{P}(Z \leq x)$ be the cumulative distribution function of an essentially bounded random variable $Z$, and let $F_{Z}^{\leftarrow}(p)=\inf \{x$ : $F_{Z}(x) \geq p$ \} be the generalized inverse of $F_{Z}$. Given a risk spectrum $\phi \in L_{1}([0,1])$, the spectral risk measure $\rho_{\phi}$ generated by $\phi$ is (see [1]):

$\rho_{\phi}(Z)=-\int_{0}^{1} F_{Z}^{\leftarrow}(p) \phi(p) d p$

Spectral risk measures have been used in various applications (portfolio selection by Acerbi and Simonetti [2]; insurance by Cotter and Dowd [4]). The conditional value-at-risk (CVaR) of level $0<\varepsilon<1$, denoted by $C V a R^{\varepsilon}$, is a particular spectral risk measure obtained taking $\phi(u)=\frac{1}{\varepsilon} 1_{0 \leq u<\varepsilon}$ (see Acerbi [1]).

In what follows, we consider more generally a piecewise constant risk function $\phi(\cdot)$ with $J$ jumps at $0<p_{1}<p_{2}<\cdots<$ $p_{J}<1$. We set $\Delta \phi_{k}=\phi\left(p_{k}^{+}\right)-\phi\left(p_{k}^{-}\right)=\phi\left(p_{k}\right)-\phi\left(p_{k-1}\right)$, for $k=$ $1, \ldots, J$, with $p_{0}=0$, and we assume that

(i) $\phi(\cdot)$ is positive, $\quad$ (ii) $\Delta \phi_{k}<0, \quad k=1, \ldots, J$,

(iii) $\int_{0}^{1} \phi(u) d u=1$.

In this context, $\rho_{\phi}$ can be expressed as a linear combination of conditional value-at-risk measures. With this choice of risk function $\phi$, the spectral risk measure $\rho_{\phi}(Z)$ can be expressed as the optimal value of a linear program; see Acerbi and Simonetti [2]:

$\rho_{\phi}(Z)=\inf _{w \in \mathbb{R}^{J}} \sum_{k=1}^{J} \Delta \phi_{k}\left[p_{k} w_{k}-\mathbb{E}\left[w_{k}-Z\right]^{+}\right]-\phi(1) \mathbb{E}[Z]$.
Using this formulation for $\rho_{\phi}$, dynamic programming equations are given in [8] for risk-averse formulation (1). More precisely, problem (1) can be expressed as

$\inf _{x_{1}, w_{2: T}} d_{1}^{\top} x_{1}+\sum_{t=2}^{T} \theta_{t} c_{1}^{\top} w_{t}+Q_{2}\left(x_{1}, \xi_{[1]}, z_{1}, w_{2}, \ldots, w_{T}\right)$,

$C_{1} x_{1}=\xi_{1}-D_{1} x_{0}, \quad x_{1} \geq 0, w_{t} \in \mathbb{R}^{J}, t=2, \ldots, T$,

with $z_{1}=0$, vector $c_{1}=\Delta \phi \circ p$, and where, for $t=2, \ldots, T$,

$Q_{t}\left(x_{t-1}, \xi_{[t-1]}, z_{t-1}, w_{t: T}\right)$

$$
=\mathbb{E}_{\xi_{t} \mid \xi_{[t-1]}}\left(\begin{array}{l}
\inf _{x_{t}, z_{t}} f_{t}\left(z_{t}, w_{t}\right)+Q_{t+1}\left(x_{t}, \xi_{[t]}, z_{t}, w_{t+1: T}\right) \\
z_{t}=z_{t-1}-d_{t}^{\top} x_{t}, C_{t} x_{t}=\xi_{t}-D_{t} x_{t-1}, x_{t} \geq 0
\end{array}\right),
$$

with

$f_{t}\left(z_{t}, w_{t}\right)=-\left(\delta_{t T} \theta_{1}+\phi(1) \theta_{t}\right) z_{t}-\theta_{t} \Delta \phi^{\top}\left(w_{t}-z_{t} e\right)^{+}$,

and $\mathcal{Q}_{T+1} \equiv 0$. Function $\mathcal{Q}_{t+1}$ represents at stage $t$ a cost-togo or recourse function which is risk averse. As shown in the next section, it can be approximated by cutting planes by some polyhedral function $\mathfrak{Q}_{t+1}$. These approximate recourse functions are useful for defining a feasible approximate policy obtained by solving

$\inf _{x_{t}, z_{t}} f_{t}\left(z_{t}, w_{t}\right)+\mathfrak{Q}_{t+1}\left(x_{t}, \xi_{[t]}, z_{t}, w_{t+1: T}\right)$

$C_{t} x_{t}=\xi_{t}-D_{t} x_{t-1}, \quad x_{t} \geq 0, z_{t}=z_{t-1}-d_{t}^{\top} x_{t}$,

at stage $t=2, \ldots, T$, knowing $x_{t-1}, z_{t-1}$, first-stage decision variables $w_{t: T}$, and $\xi_{t}$. First-stage decision variables $x_{1}$ and $w_{2: T}$ are the solution to (3) with $\mathcal{Q}_{2}$ replaced by the approximation $\mathfrak{Q}_{2}$.

\section{Algorithmic issues}

The DP equations (3)-(4) make possible the use of decomposition algorithms such as SDDP to obtain approximations of the corresponding recourse functions. When applied to DP equations (3)-(4), the convergence of this algorithm is proved in [8] under the following assumptions.

(A1) The supports of the distributions of $\xi_{1}, \ldots, \xi_{T}$ are discrete and finite.

(A2) Process $\left(\xi_{t}\right)$ is interstage independent.

(A3) For $t=1, \ldots, T$, for any feasible $x_{t-1}$, and for any realization $\tilde{\xi}_{t}$ of $\xi_{t}$, the set

$\left\{x_{t}: x_{t} \geq 0, C_{t} x_{t}=\tilde{\xi}_{t}-D_{t} x_{t-1}\right\}$

is bounded and nonempty.

In what follows, we assume that Assumptions (A1)-(A3) hold. In particular, we denote the realizations of $\xi_{t}$ by $\xi_{t}^{i}, i=1, \ldots, q_{t}<$ $+\infty$, and set $p(t, i)=\mathbb{P}\left(\xi_{t}=\xi_{t}^{i}\right)$.

Since the supports of the distributions of the random vectors $\xi_{2}, \ldots, \xi_{T}$ are discrete and finite, optimization problem (1) is finite dimensional, and the evolution of the uncertain parameters over the optimization period can be represented by a scenario tree having a finite number of scenarios that can happen in the future for $\xi_{2}, \ldots, \xi_{T}$. The root node of the scenario tree corresponds to the first time step with $\xi_{1}$ deterministic.

For a given stage $t$, to each node of the scenario tree there corresponds an history $\xi_{[t]}$. The children nodes of a node at stage $t \geq 1$ are the nodes that can happen at stage $t+1$ if we are at this node at $t$. A sampled scenario $\left(\xi_{1}, \ldots, \xi_{T}\right)$ corresponds to a particular succession of nodes such that $\xi_{t}$ is a possible value for the process at $t$ and $\xi_{t+1}$ is a child of $\xi_{t}$. A given node in the tree at stage $t$ is identified with a scenario $\left(\xi_{1}, \ldots, \xi_{t}\right)$ going from the root node to this node.

In this context, the SDDP algorithm builds polyhedral lower bounding approximations $\mathfrak{Q}_{t}$ of $\mathcal{Q}_{t}$ for $t=2, \ldots, T+1$. Each 
iteration of this algorithm is made of a forward pass followed by a backward pass. Approximation $\mathfrak{Q}_{t}^{i}$ for $\mathcal{Q}_{t}$ available at the end of iteration $i$ can be expressed as a maximum of cuts (hyperplanes lying below the recourse functions) built in the backward passes:

$$
\begin{aligned}
\mathfrak{Q}_{t}^{i}\left(x_{t-1}, z_{t-1}, w_{t: T}\right)= & \max _{j=0,1, \ldots, i H}\left[-E_{t-1}^{j} x_{t-1}-Z_{t-1}^{j} z_{t-1}\right. \\
& \left.+\sum_{\tau=1}^{T-t+1} W_{t-1}^{j, \tau} w_{t+\tau-1}+e_{t-1}^{j}\right],
\end{aligned}
$$

knowing that the algorithm starts taking for $\mathfrak{Q}_{t}^{0}$ a known lower bounding affine approximation of $\mathcal{Q}_{t}$ while $\mathfrak{Q}_{T+1}^{i} \equiv 0$. In the above expression, we have assumed that $H$ cuts are built at each iteration. If the algorithm runs for $K$ iterations, we end up with approximate recourse functions $\mathfrak{Q}_{t}=\mathfrak{Q}_{t}^{K}, t=2, \ldots, T+1$.

At iteration $i$, cuts for $\mathcal{Q}_{t}, t=2, \ldots, T$, are built at some points $x_{t-1}^{k}, z_{t-1}^{k}, w_{t: T}^{i}, k=(i-1) H+1, \ldots, i H$, computed in the forward pass replacing the recourse functions $\mathcal{Q}_{t+1}$ by $\mathfrak{Q}_{t+1}^{i-1}$ (note that, since variables $w_{2: T}$ are first-stage decision variables, they just depend on the iteration).

More precisely, the cuts are computed for time step $T+1$ down to time step 2. For time step $T+1$, since $\mathfrak{Q}_{T+1}^{i}=\mathcal{Q}_{T+1}$ $=0$, the cuts for $Q_{T+1}$ are obtained taking null vectors for $E_{T}^{k}, Z_{T}^{k}$, $W_{T}^{k, \tau}$, and $e_{T}^{k}$ for $k=(i-1) H+1, \ldots, i H$. For $t=2, \ldots, T$, using the lower bounding approximation $\mathfrak{Q}_{t+1}^{i}$ of $\mathcal{Q}_{t+1}$, we can bound from below $\mathcal{Q}_{t}\left(x_{t-1}, z_{t-1}, w_{t: T}\right)$ by $\mathbb{E}_{\xi_{t}}\left[Q_{t}^{i}\left(x_{t-1}, z_{t-1}, w_{t: T}, \xi_{t}\right)\right]$ with $Q_{t}^{i}\left(x_{t-1}, z_{t-1}, w_{t: T}, \xi_{t}\right)$ given as the optimal value of the following linear program:

$$
\begin{aligned}
& \quad \inf _{x_{t}, z_{t}, v_{t}, \tilde{\theta}_{t}}-\left(\delta_{t \top} \theta_{1}+\phi(1) \theta_{t}\right) z_{t}-\theta_{t} \Delta \phi^{\top} v_{t}+\tilde{\theta}_{t} \\
& v_{t} \geq 0, \quad v_{t} \geq w_{t}-z_{t} e, \quad x_{t} \geq 0, \\
& z_{t}+d_{t}^{\top} x_{t}=z_{t-1} \\
& C_{t} x_{t}=\xi_{t}-D_{t} x_{t-1} \\
& \vec{E}_{t}^{i} x_{t}+\vec{Z}_{t}^{i} z_{t}+\tilde{\theta}_{t} e \geq \sum_{\tau=1}^{T-t} \vec{W}_{t}^{i, \tau} w_{t+\tau}+\vec{e}_{t}^{i},
\end{aligned}
$$

where $\vec{E}_{t}^{i}$ (respectively, $\vec{Z}_{t}^{i}, \vec{W}_{t}^{i, \tau}$, and $\vec{e}_{t}^{i}$ ) is the matrix whose $(j+1)$ th line is $E_{t}^{j}$ (respectively, $Z_{t}^{j}, W_{t}^{j, \tau}$, and $e_{t}^{j}$ ) for $j=$ $0, \ldots, i H$. In the backward pass of iteration $i$, the above problem is solved with $\left(x_{t-1}, z_{t-1}, w_{t: T}, \xi_{t}\right)$ respectively replaced by $\left(x_{t-1}^{k}, z_{t-1}^{k}, w_{t: T}^{i}, \xi_{t}^{j}\right)$ for $k=(i-1) H+1, \ldots, i H$ and $j=$ $1, \ldots, q_{t}$. Let $\sigma_{t}^{k, j}, \tilde{\sigma}_{t}^{k, j}, \mu_{t}^{k, j}, \pi_{t}^{k, j}$, and $\rho_{t}^{k, j}$ be the (row vectors) optimal Lagrange multipliers respectively for the constraints $v_{t} \geq$ $w_{t}^{i}-z_{t} e, v_{t} \geq 0,(8 \mathrm{a}),(8 \mathrm{~b})$ and (8c) for the problem defining $Q_{t}^{i}\left(x_{t-1}^{k}, z_{t-1}^{k}, w_{t: T}^{i}, \xi_{t}^{j}\right)$ for $k=(i-1) H+1, \ldots, i H$ and $j=$ $1, \ldots, q_{t}$. The following proposition provides the cuts computed for $Q_{t}, t=2, \ldots, T$, at iteration $i$.

Proposition 3.1 (Optimality Cuts). Let $Q_{t}, t=2, \ldots, T+1$, be the risk-averse recourse functions given by (4). In the backward pass of iteration $i$ of the SDDP algorithm, the following cuts are computed for these recourse functions. For $t=T+1, E_{t-1}^{k}, Z_{t-1}^{k}, W_{t-1}^{k, \tau}$, and $e_{t-1}^{k}$ are null for $k=(i-1) H+1, \ldots, i H$. For $t=2, \ldots, T$ and $k=(i-1) H+1, \ldots, i H, E_{t-1}^{k}$ is given by $\sum_{j=1}^{q_{t}} p(t, j) \pi_{t}^{k, j} D_{t}$, and

$$
\begin{aligned}
Z_{t-1}^{k} & =-\sum_{j=1}^{q_{t}} p(t, j) \mu_{t}^{k, j}, \quad W_{t-1}^{k, 1}=\sum_{j=1}^{q_{t}} p(t, j) \sigma_{t}^{k, j} \\
W_{t-1}^{k, \tau} & =\sum_{j=1}^{q_{t}} p(t, j) \rho_{t}^{k, j} \vec{W}_{t}^{i, \tau-1}, \quad \tau=2, \ldots, T-t+1 .
\end{aligned}
$$

Further, $e_{t-1}^{k}$ is given by

$$
\begin{aligned}
& \sum_{j=1}^{q_{t}} p(t, j)\left[Q_{t}^{i}\left(x_{t-1}^{k}, z_{t-1}^{k}, w_{t: T}^{i}, \xi_{t}^{j}\right)-\mu_{t}^{k, j} z_{t-1}^{k}\right. \\
& \left.\quad-\sigma_{t}^{k, j} w_{t}^{i}-\sum_{\tau=1}^{T-t} \rho_{t}^{k, j} \vec{W}_{t}^{i, \tau} w_{t+\tau}^{i}+\pi_{t}^{k, j} D_{t} x_{t-1}^{k}\right] .
\end{aligned}
$$

Proof. Since a dual solution of the problem defining $Q_{t}^{i}\left(x_{t-1}^{k}\right.$, $\left.z_{t-1}^{k}, w_{t: T}^{i}, \xi_{t}^{j}\right)$ is a subgradient of the value function for problem (8), we obtain that $Q_{t}^{i}\left(x_{t-1}, z_{t-1}, w_{t: T}, \xi_{t}^{j}\right)$ is bounded from below by

$$
\begin{aligned}
& Q_{t}^{i}\left(x_{t-1}^{k}, z_{t-1}^{k}, w_{t: T}^{i}, \xi_{t}^{j}\right)+\mu_{t}^{k, j}\left(z_{t-1}-z_{t-1}^{k}\right)+\sigma_{t}^{k, j}\left(w_{t}-w_{t}^{i}\right) \\
& \quad+\sum_{\tau=2}^{T-t+1} \rho_{t}^{k, j} \vec{W}_{t}^{i, \tau-1}\left(w_{t+\tau-1}-w_{t+\tau-1}^{i}\right) \\
& \quad-\pi_{t}^{k, j} D_{t}\left(x_{t-1}-x_{t-1}^{k}\right) .
\end{aligned}
$$

Using the above lower bound and the fact that $Q_{t}\left(x_{t-1}, z_{t-1}, w_{t: T}\right)$ is bounded from below by $\sum_{j=1}^{q_{t}} p(t, j) Q_{t}^{i}\left(x_{t-1}, z_{t-1}, w_{t: T}, \xi_{t}^{j}\right)$, we obtain the announced cuts.

The stopping criterion is discussed in [22] for a non-risk-averse model. The definition of a sound stopping criterion for the riskaverse model from [22] (based on a nested formulation of the problem defined in terms of conditional risk mappings) is a more delicate issue, and is still open for discussion. However, since problem (1) can be expressed as a non-risk-averse problem with modified objective, variables, and constraints, in our riskaverse context the stopping criterion is a simple adaptation of the stopping criterion for the non-risk-averse case.

More specifically, in the backward pass of iteration $i$, for the first time step, first-stage problem (3) is solved by replacing the recourse function $Q_{2}$ by $\mathfrak{Q}_{2}^{i} \leq Q_{2}$. As a result, the optimal value of this problem gives a lower bound $z_{\text {inf }}$ on the optimal value of (1).

In the forward pass of iteration $i$, we can compute the total cost $\mathcal{C}_{k}$ on each scenario $k=(i-1) H+1, \ldots, i H$ :

$\mathcal{C}_{k}=d_{1}^{\top} x_{1}^{k}+\sum_{t=2}^{T} \theta_{t} c_{1}^{\top} w_{t}^{i}+\sum_{t=2}^{T} f_{t}\left(z_{t}^{k}, w_{t}^{i}\right)$.

If these $H$ scenarios were representing all possible evolutions of $\left(\xi_{1}, \ldots, \xi_{T}\right)$, then

$\overline{\mathrm{C}}=\frac{1}{H} \sum_{k=(i-1) H+1}^{i H} \mathcal{C}_{k}$

would be an upper bound on the optimal value of (1) (recall that the approximate policy is feasible and that the objective function of (1) can be written as an expectation). Since we only have a sample of all the possible scenarios, $\overline{\mathcal{C}}$ is an estimation of an upper bound on this optimal value. Introducing the empirical standard deviation $\bar{\sigma}$ of the sample $\left(\mathcal{C}_{1}, \ldots, \mathcal{C}_{H}\right)$,

$\bar{\sigma}=\sqrt{\frac{1}{H-1} \sum_{k=(i-1) H+1}^{i H}\left(\overline{\mathrm{C}}-\mathcal{C}_{k}\right)^{2},}$

we can compute the $(1-\alpha)$-confidence upper bound

$\bar{\complement}+t_{1-\alpha, H-1} \frac{\bar{\sigma}}{\sqrt{H}}$

on the approximate policy mean value, where $t_{1-\alpha, H-1}$ is the (1$\alpha$ )-quantile of the Student $t$-distribution with $H-1$ degrees of freedom. Since the optimal value of (1) is less than or equal to the 
Step 0: INITIALIZATION. Set $i=1$ (iteration number) and select confidence levels $\alpha \in(1 / 2,1)$ and $\varepsilon>0$. Take null values for $E_{t-1}^{0}, Z_{t-1}^{0}, W_{t-1}^{0, \tau}, t=2, \ldots, T+1$. Take $e_{T}^{0}=0$ and for $e_{t-1}^{0}$ a lower bound on $\mathcal{Q}_{t}$ for $t=2, \ldots, T$. Go to Step 1 .

Step 1: FORWARD PASS.

Sample $H$ scenarios $\left(\xi_{1}, \xi_{2}^{k}, \ldots, \xi_{T}^{k}\right), k=(i-1) H+1, \ldots, i H$.

$\mathrm{Ct}=0, \mathrm{Ct}_{-} \mathrm{Sq}=0$.

Solve the first stage problem

$$
\begin{aligned}
& \inf _{x_{1}, w_{2: T}} d_{1}^{\top} x_{1}+\sum_{t=2}^{T} \theta_{t} c_{1}^{\top} w_{t}+\mathfrak{Q}_{2}^{i-1}\left(x_{1}, z_{1}, w_{2}, \ldots, w_{T}\right), \\
& C_{1} x_{1}=\xi_{1}-D_{1} x_{0}, x_{1} \geq 0, w_{t} \in \mathbb{R}^{J}, t=2, \ldots, T,
\end{aligned}
$$

and store an optimal solution $\left(x_{1}^{*}, w_{2: T}^{i}\right)$.

For $k=(i-1) H+1, \ldots, i H$,

Set $x_{1}^{k}=x_{1}^{*}$.

For $t=2, \ldots, T$,

Solve

$$
\begin{aligned}
& \inf _{x_{t}, z_{t}} f_{t}\left(z_{t}, w_{t}^{i}\right)+\mathfrak{Q}_{t+1}^{i-1}\left(x_{t}, z_{t}, w_{t+1: T}^{i}\right) \\
& C_{t} x_{t}=\xi_{t}^{k}-D_{t} x_{t-1}^{k}, x_{t} \geq 0, z_{t}=z_{t-1}^{k}-d_{t}^{\top} x_{t},
\end{aligned}
$$

\section{End For}

and store an optimal solution $\left(x_{t}^{k}, z_{t}^{k}\right)$.

Compute $\mathcal{C}_{k}$ given by (11),

$\mathrm{Ct}=\mathrm{Ct}+\mathcal{C}_{k}, \mathrm{Ct}_{-} \mathrm{Sq}=\mathrm{Ct}_{-} \mathrm{Sq}+\mathcal{C}_{k}^{2}$.

End For

Step 2: BACKWARD PASS.

$\overline{\mathcal{C}}=\frac{\mathrm{Ct}}{H}, \bar{\sigma}=\sqrt{\frac{1}{H-1}\left(\mathrm{Ct} \_\mathrm{Sq}-H \overline{\mathcal{C}}^{2}\right)}, z_{\text {sup }}=\overline{\mathcal{C}}+t_{1-\alpha, H-1} \frac{\bar{\sigma}}{\sqrt{H}}$. Go to Step 2 .

For $t=T+1$ down to 2 ,

For $k=(i-1) H+1, \ldots, i H$,

If $(t=T+1)$ then set $E_{t-1}^{k}, Z_{t-1}^{k}, W_{t-1}^{k, \tau}$, and $e_{t-1}^{k}$ to 0 .

Else

$$
\begin{aligned}
& \text { For } j=1, \ldots, q_{t}, \\
& \quad \text { Compute } Q_{t}^{i}\left(x_{t-1}^{k}, z_{t-1}^{k}, w_{t: T}^{i}, \xi_{t}^{j}\right) \text {, i.e., solve (8) replacing } \\
& \quad\left(x_{t-1}, z_{t-1}, w_{t: T}, \xi_{t}\right) \text { by }\left(x_{t-1}^{k}, z_{t-1}^{k}, w_{t: T}^{i}, \xi_{t}^{j}\right) \text { and } \\
& \text { store a dual solution. }
\end{aligned}
$$

End For

Build a cut for $\mathcal{Q}_{t}$, i.e., compute $E_{t-1}^{k}, Z_{t-1}^{k}, W_{t-1}^{k, \tau}$, and $e_{t-1}^{k}$

End If

using the formulas from Proposition 3.1.

\section{End For}

\section{End For}

Set $z_{\text {inf }}$ to the optimal value of the first stage problem.

Go to Step 3.

\section{Step 3: STOPPING RULE.}

If $z_{\text {sup }}-z_{\text {inf }} \leq \varepsilon$ then stop.

Else $i \leftarrow i+1$ and go to Step 1. End If

Fig. 1. SDDP algorithm with relatively complete recourse for the risk-averse interstage independent stochastic linear program (1).

approximate policy mean value, (12) gives an upper bound for the optimal value of $(1)$ with confidence at least $1-\alpha$. Consequently, we can stop the algorithm when $\overline{\mathcal{C}}+t_{1-\alpha, H-1} \frac{\bar{\sigma}}{\sqrt{H}}-z_{\text {inf }} \leq \varepsilon$ for some $\varepsilon>0$.

Using the previous developments, the SDDP algorithm for solving (1) can be formulated as in Fig. 1.

We now give, for some particular choices of the first-stage variables $w_{2: T}^{1}$, the exact expressions (independent of the sampled scenarios) of $Z_{t-1}^{k}$ and $W_{t-1}^{k, \tau}$ for every $t=2, \ldots, T, k=1, \ldots, H$, and $\tau=1, \ldots, T-t+1$. Though the first-stage feasible set for (3) is not bounded, it can be easily shown that the optimal values of $w_{2: T}$ are bounded (see [8], for instance). As a result, well-chosen box constraints on $w_{t}, t=2, \ldots, T$ can be added (at the first stage, and that do not modify the optimal value of (3)) without changing the cut calculations (since these latter are performed for stages $t=2, \ldots, T$, where $w_{t}$ are state variables).

Let us define, for $t=1, \ldots, T, x^{t}=\left(x_{1}, \ldots, x_{t}\right), \xi^{t}=$ $\left(\xi_{1}, \ldots, \xi_{t}\right)$, and let us introduce the set $\chi^{t}$ of admissible decisions up to time step $t$ :

$$
\begin{aligned}
\chi^{t}= & \left\{x^{t}: \exists \tilde{\xi}^{t} \text { realization of } \xi^{t}: x_{\tau} \geq 0\right. \\
& \text { and } \left.C_{\tau} x_{\tau}=\tilde{\xi}_{\tau}-D_{\tau} x_{\tau-1}, \tau=1, \ldots, t\right\} .
\end{aligned}
$$


Since (A3) holds, the sets $\chi^{t}$ are compact, and, since $g^{t}\left(x^{t}\right)=$ $\sum_{\tau=2}^{t} d_{\tau}^{\top} x_{\tau}$ is continuous, we can introduce the pairs $\left(C_{t}^{u}, C_{t}^{\ell}\right) \in \mathbb{R}^{2}$ defined by

$C_{t}^{u}=\left\{\begin{array}{l}\max g^{t}\left(x^{t}\right) \\ x^{t} \in \chi^{t},\end{array} \quad C_{t}^{\ell}=\left\{\begin{array}{l}\min g^{t}\left(x^{t}\right) \\ x^{t} \in \chi^{t} .\end{array}\right.\right.$

The objective of the forward pass is to build states where cuts are computed in the backward pass. At the first iteration, instead of building these states using the approximate recourse functions $\mathfrak{Q}_{t}^{0}$, we can choose arbitrary feasible states $x_{t-1}^{k}, z_{t-1}^{k}, w_{t}^{1}, t=$ $2, \ldots, T$ (which is a simple task, since relatively complete recourse holds). With this variant of the first iteration, we have $i H$ cuts for $\mathfrak{Q}_{t}^{i}$ at the end of iteration $i$. If we choose first-stage variables $w_{2: T}^{1}$ such that (i) $w_{t}^{1}>-C_{t}^{\ell} e$ for $t=2, \ldots, T$ (respectively, such that (ii) $w_{t}^{1}<-C_{t}^{u} e$ for $\left.t=2, \ldots, T\right)$, then $Z_{t-1}^{k}$ and $W_{t-1}^{k, \tau}$, for $k=$ $1, \ldots, H$, can be computed using Proposition 3.2(i) (respectively, Proposition 3.2(ii)), which follows. For instance, if the costs are positive, then item (i) is fulfilled with $w_{t}^{1}=0$ and item (ii) by taking for each component of $w_{t}^{1}$ the opposite of a strict upper bound on the worst cost.

Proposition 3.2 (Cuts Calculation at the First Iteration). Let us consider the risk-averse recourse functions $\mathcal{Q}_{t}$ given by (4). Valid cuts for $\mathcal{Q}_{t}$ are given by Proposition 3.1. Moreover, in the following two cases, we have closed-form expressions for $Z_{t-1}^{k}$ and $W_{t-1}^{k, \tau}$ (independent of the sampled scenarios).

(i) If, for $t=2, \ldots, T, w_{t}^{1}>-C_{t}^{\ell} e$, then, for $t=2, \ldots, T, \mathcal{P}(t)$ holds, where

$$
\mathcal{P}(t):\left\{\begin{array}{c}
\forall k=1, \ldots, H, \quad Z_{t-1}^{k}=\theta_{1}+\phi(0) \sum_{\ell=t}^{T} \theta_{\ell}, \\
\forall k=1, \ldots, H, \quad W_{t-1}^{k, \tau}=-\theta_{t+\tau-1} \Delta \phi^{\top} \\
\tau=1, \ldots, T-t+1
\end{array}\right.
$$

(ii) If, for $t=2, \ldots, T, w_{t}^{1}<-C_{t}^{u} e$, then, for $t=2, \ldots, T, \tilde{\mathcal{P}}(t)$ holds, where

$$
\tilde{\mathcal{P}}(t): \begin{cases}\forall k=1, \ldots, H, & Z_{t-1}^{k}=\theta_{1}+\phi(1) \sum_{\ell=t}^{T} \theta_{\ell}, \\ \forall k=1, \ldots, H, & W_{t-1}^{k, \tau}=0 . \\ \forall \tau=1, \ldots, T-t+1, & \end{cases}
$$

Proof. Let us fix $t \in\{2, \ldots, T\}, k \in\{1, \ldots, H\}$, and $j \in\{1, \ldots$, $\left.q_{t}\right\}$. We denote by $x_{t}, z_{t}, v_{t}, \tilde{\theta}_{t}$ an optimal solution to the problem defining $Q_{t}^{1}\left(x_{t-1}^{k}, z_{t-1}^{k}, w_{t: T}^{1}, \xi_{t}^{j}\right)$, i.e., problem (8) written for $i=1$, and with $\left(x_{t-1}, z_{t-1}, w_{t: T}, \xi_{t}\right)$ replaced by $\left(x_{t-1}^{k}, z_{t-1}^{k}, w_{t: T}^{i}, \xi_{t}^{j}\right)$ (the dependence of the solution with respect to $k, j$ is suppressed, to alleviate notation).

The Karush-Kuhn-Tucker (KKT) conditions for this problem imply that

$-\delta_{t T} \theta_{1}-\phi(1) \theta_{t}-\mu_{t}^{k, j}-\sigma_{t}^{k, j} e-\rho_{t}^{k, j} \vec{Z}_{t}^{1}=0$,

$-\theta_{t} \Delta \phi^{\top}-\tilde{\sigma}_{t}^{k, j}-\sigma_{t}^{k, j}=0$,

$\sigma_{t}^{k, j} \circ\left(-z_{t} e+w_{t}^{1}-v_{t}\right)^{\top}=0$,

$\tilde{\sigma}_{t}^{k, j} \circ v_{t}^{\top}=0$,

where, for $t=T$, we have set $\rho_{t}^{k, j}=0$. Next, since $z_{t}$ can be written as $z_{t}=-g^{t}\left(x^{t}\right)$ for some $x^{t} \in \chi^{t}$, in case (i), we have $z_{t} e \leq-C_{t}^{\ell} e<w_{t}^{1}$. Further, $v_{t}=\max \left(0, w_{t}^{1}-z_{t} e\right)=w_{t}^{1}-z_{t} e>0$.
Using (14) and (16), we then get

$\tilde{\sigma}_{t}^{k, j}=0$ and $\sigma_{t}^{k, j}=-\theta_{t} \Delta \phi^{\top}$.

Let us now first show (i) by backward induction on $t$. Plugging the value of $\sigma_{T}^{k, j}$ given in (17) into (13), we obtain

$$
\begin{aligned}
\mu_{T}^{k, j} & =-\theta_{1}-\phi(1) \theta_{T}+\theta_{T} e^{\top} \Delta \phi \\
& =-\theta_{1}+\theta_{T}\left(-\phi(1)+\sum_{\ell=1}^{J}\left[\phi\left(p_{\ell}\right)-\phi\left(p_{\ell-1}\right)\right]\right) \\
& =-\theta_{1}-\theta_{T} \phi(0) .
\end{aligned}
$$

Using the above relation and (9) yields $Z_{T-1}^{k}=-\sum_{j=1}^{q_{T}} p(T, j) \mu_{T}^{k, j}$ $=\theta_{T} \phi(0)+\theta_{1}$. Further, using once again (9), we obtain

$$
\begin{aligned}
W_{T-1}^{k, 1} & =\sum_{j=1}^{q_{T}} p(T, j) \sigma_{T}^{k, j} \\
& =-\sum_{j=1}^{q_{T}} p(T, j) \theta_{T} \Delta \phi^{\top}=-\theta_{T} \Delta \phi^{\top} .
\end{aligned}
$$

This shows $\mathcal{P}(T)$. Let us now assume that $\mathcal{P}(t+1)$ holds for some $t \in\{2, \ldots, T-1\}$, and let us show that $\mathcal{P}(t)$ holds. First, notice that (18) still holds with $T$ substituted with $t$, i.e., $W_{t-1}^{k, 1}=-\theta_{t} \Delta \phi^{\top}$. Further, for $\tau=2, \ldots, T-t+1$,

$$
\begin{aligned}
W_{t-1}^{k, \tau} & =\sum_{j=1}^{q_{t}} p(t, j) \rho_{t}^{k, j} \vec{W}_{t}^{1, \tau-1}, \quad \text { from }(10), \\
& =-\sum_{j=1}^{q_{t}} p(t, j) \rho_{t}^{k, j} \theta_{t+\tau-1} e \Delta \phi^{\top}, \quad \text { using } \mathcal{P}(t+1), \\
& =-\sum_{j=1}^{q_{t}} p(t, j) \theta_{t+\tau-1} \Delta \phi^{\top} \quad \text { since } \rho_{t}^{k, j} e=1, \\
& =-\theta_{t+\tau-1} \Delta \phi^{\top} .
\end{aligned}
$$

Also,

$$
\begin{aligned}
Z_{t-1}^{k}= & -\sum_{j=1}^{q_{t}} p(t, j) \mu_{t}^{k, j}, \quad \text { from }(9) \\
= & -\sum_{j=1}^{q_{t}} p(t, j)\left(-\phi(1) \theta_{t}+\theta_{t} \Delta \phi^{\top} e-\rho_{t}^{k, j} \vec{Z}_{t}^{1}\right), \\
& \text { using }(13) \text { and }(17), \\
= & -\sum_{j=1}^{q_{t}} p(t, j)\left(-\phi(0) \theta_{t}-\rho_{t}^{k, j} \vec{Z}_{t}^{1}\right), \\
& \text { using the definition of } \Delta \phi, \\
= & \phi(0) \theta_{t}+\sum_{j=1}^{q_{t}} p(t, j) \rho_{t}^{k, j}\left(\theta_{1}+\phi(0) \sum_{\ell=t+1}^{T} \theta_{\ell}\right) e \\
& \operatorname{using} \mathcal{P}(t+1), \\
= & \theta_{1}+\phi(0) \sum_{\ell=t}^{T} \theta_{\ell} \quad \text { since } \rho_{t}^{k, j} e=1 .
\end{aligned}
$$

We have thus shown $\mathcal{P}(t)$ which achieves the proof of (i).

Let us now assume that $w_{t}^{1}<-C_{t}^{u} e$ for $t=2, \ldots, T$, and let us show (ii). Let us fix $t \in\{2, \ldots, T\}, k \in\{1, \ldots, H\}$, and $j \in\left\{1, \ldots, q_{t}\right\}$. As before, we denote by $x_{t}, z_{t}, v_{t}, \tilde{\theta}_{t}$ an optimal solution to the problem defining $Q_{t}^{1}\left(x_{t-1}^{k}, z_{t-1}^{k}, w_{t: T}^{1}, \xi_{t}^{j}\right)$. In this case, $z_{t} e \geq-C_{t}^{u} e>w_{t}^{1}$ and $v_{t}=\max \left(0, w_{t}^{1}-z_{t} e\right)=0$. Using 
(14) and (15), we see that

$\tilde{\sigma}_{t}^{k, j}=-\theta_{t} \Delta \phi^{\top}$ and $\sigma_{t}^{k, j}=0$.

Using (9), we get $W_{t-1}^{k, 1}=0$. We show (ii) by backward induction. For $t=T$, plugging the value of $\sigma_{T}^{k, j}$ into (13) gives $\mu_{T}^{k, j}=-\theta_{1}-$ $\phi(1) \theta_{T}$, which, together with (9), gives $Z_{T-1}^{k}=\theta_{1}+\phi(1) \theta_{T}$. We have already proved that $W_{T-1}^{k, 1}=0$, and thus $\tilde{\mathcal{P}}(T)$ holds. Let us now assume that $\mathcal{P}(t+1)$ holds for some $t \in\{2, \ldots, T-1\}$, and let us show that $\mathcal{P}(t)$ holds. Since $\vec{W}_{t}^{1, \tau-1}=0$, we obtain $W_{t-1}^{k, \tau}=\sum_{j=1}^{q_{t}} p(t, j) \rho_{t}^{k, j} \vec{W}_{t}^{1, \tau-1}=0$ for $\tau=2, \ldots, T-t+1$. Plugging $\sigma_{t}^{k, j}=0$ into (13) and using (9) gives

$$
\begin{aligned}
Z_{t-1}^{k}= & \sum_{j=1}^{q_{t}} p(t, j)\left(\phi(1) \theta_{t}+\rho_{t}^{k, j} \vec{Z}_{t}^{1}\right), \\
= & \sum_{j=1}^{q_{t}} p(t, j)\left(\theta_{1}+\phi(1) \sum_{\ell=t}^{T} \theta_{\ell}\right), \\
& \quad \text { using } \tilde{\mathcal{P}}(t+1) \quad \text { and } \rho_{t}^{k, j} e=1, \\
= & \theta_{1}+\phi(1) \sum_{\ell=t}^{T} \theta_{\ell} .
\end{aligned}
$$

This shows $\tilde{\mathcal{P}}(t)$ and achieves the proof of (ii).

Proposition 3.2 can be used as a debugging tool to check the implementation of the SDDP algorithm for risk-averse problem (1). More precisely, we can check that, in cases (i) and (ii), implementing the formulas for $Z_{t-1}^{k}$ and $W_{t-1}^{k, \tau}$ given in Proposition 3.1 will give the same results as implementing the formulas from Proposition 3.2.

At stage $t$, if instead of $\rho_{\phi}$ in (1) we use $C V a R^{\varepsilon t}$, problem (1) becomes

$\inf _{x_{1}, \ldots, x_{T}} d_{1}^{\top} x_{1}+\theta_{1} \mathbb{E}\left[\sum_{t=2}^{T} d_{t}^{\top} x_{t}\right]+\sum_{t=2}^{T} \theta_{t} C \operatorname{VaR}^{\varepsilon t}\left(-\sum_{k=2}^{t} d_{k}^{\top} x_{k}\right)$

$C_{t} x_{t}=\xi_{t}-D_{t} x_{t-1}$

$x_{t} \geq 0, \quad x_{t}$ is $\mathscr{F}_{t}$-measurable, $t=1, \ldots, T$.

For this model, we obtain a result analogous to Proposition 3.2.

Proposition 3.3. Let us consider the risk-averse recourse functions $Q_{t}$ for model (20) and their approximations $\mathfrak{Q}_{t}^{i}$ of form (7), obtained by applying the SDDP algorithm to the corresponding DP equations. In the following two cases, we obtain closed-form expressions for $Z_{t-1}^{k}$ and $W_{t-1}^{k, \tau}$ (independent of the sampled scenarios).

(i) If, for $t=2, \ldots, T, w_{t}^{1}>-C_{t}^{\ell}$, then, for $t=2, \ldots, T, \mathcal{P}(t)$ holds, where

$$
\mathcal{P}(t): \begin{cases}\forall k=1, \ldots, H, & Z_{t-1}^{k}=\theta_{1}+\sum_{\ell=t}^{T} \frac{\theta_{\ell}}{\varepsilon_{\ell}}, \\ \forall k=1, \ldots, H, & W_{t-1}^{k, \tau}=\frac{\theta_{t+\tau-1}}{\varepsilon_{t+\tau-1}} . \\ \forall \tau=1, \ldots, T-t+1, & \end{cases}
$$

(ii) If, for $t=2, \ldots, T, w_{t}^{1}<-C_{t}^{u}$, then, for $t=2, \ldots, T$, $\tilde{\mathcal{P}}(t)$ holds, where

$$
\begin{array}{lll}
\tilde{\mathcal{P}}(t): & \forall k=1, \ldots, H, & Z_{t-1}^{k}=\theta_{1}, \quad \text { and } \\
& \forall \tau=1, \ldots, T-t+1, & W_{t-1}^{k, \tau}=0 .
\end{array}
$$

Proof. The proof is similar to the proof of Proposition 3.2

Remark 3.4. In the particular case when the CVaR levels $\varepsilon_{t}=$ $\varepsilon \in(0,1)$ are the same at each time step, Proposition 3.3 is a particular case of Proposition 3.2 with $\phi(1)=0, \phi(0)=\frac{1}{\varepsilon}$, and $\Delta \phi=-1 / \varepsilon \in \mathbb{R}$.

Numerical simulations for a real-life application modeled as (20) are reported in [7].

When Assumption (A1) does not hold, as stated in [22], a feasible nonanticipative policy can still be proposed using approximate recourse functions $\mathfrak{Q}_{t}$ obtained applying the SDDP algorithm on a sample average approximation (SAA) of the original problem (1)

\section{References}

[1] C. Acerbi, Spectral measures of risk: a coherent representation of subjective risk aversion, Journal of Banking and Finance 7 (2002) 1505-1518.

[2] C. Acerbi, P. Simonetti, Portfolio optimization with spectral measures of risk, Abaxbank internal report, 2002. Available at http://www.gloriamundi.org/.

[3] M. Bertocchi, G. Consigli, M.A.H. Dempster (Eds.), Stochastic Optimization Methods in Finance and Energy, Springer, New York, 2011.

[4] J. Cotter, K. Dowd, Extreme spectral risk measures: an application to futures clearinghouse variation margin requirements, Journal of Banking and Finance 30 (2006) 3469-3485.

[5] J. Dupačová, G. Consigli, S.W. Wallace, Scenarios for multistage stochastic programs, Annals of Operations Research 100 (2000) 25-53.

[6] A. Eichhorn, W. Römisch, Polyhedral risk measures in stochastic programming, SIAM Journal on Optimization 16 (2005) 69-95

[7] V. Guigues, SDDP for some interstage dependent risk-averse problems and application to hydro-thermal planning, Computational Optimization and Applications, 2011 (Submitted for publication).

[8] V. Guigues, W. Römisch, Sampling-based decomposition methods for multistage stochastic programs based on extended polyhedral risk measures, SIAM Journal on Optimization 22 (2012) 286-312.

[9] V. Guigues, C. Sagastizábal, The value of rolling horizon policies for risk averse hydro-thermal planning, European Journal of Operational Research 217 (2012) 129-140

[10] H. Heitsch, W. Römisch, Scenario tree modeling for multistage stochastic programs, Mathematical Programming 118 (2009) 371-406.

[11] H. Heitsch, W. Römisch, C. Strugarek, Stability of multistage stochastic programs, SIAM Journal on Optimization 17 (2006) 511-525.

[12] T. Pennanen, Epi-convergent discretizations of multistage stochastic programs via integration quadratures, Mathematical Programming 116 (2009) 461-479.

[13] M.V.F. Pereira, L.M.V.G Pinto, Multi-stage stochastic optimization applied to energy planning, Mathematical Programming 52 (1991) 359-375.

[14] G.Ch. Pflug, On distortion functionals, Statistics and Decisions 24 (2006) $45-60$.

[15] G.Ch. Pflug, W. Römisch, Modeling, Measuring, and Managing Risk, World Scientific, Singapore, 2007.

[16] A.B. Philpott, Z. Guan, On the convergence of stochastic dual dynamic programming and related methods, Operations Research Letters 36 (2008) $450-455$.

[17] W.B. Powell, Approximate Dynamic Programming - Solving the Curses of Dimensionality, 2nd edition, Wiley, Hoboken, New Jersey, 2011.

[18] W. Römisch, Scenario generation, in: J.J. Cochran (Ed.), Encyclopedia of Operations Research and Management Science, Wiley, 2010.

[19] A. Ruszczyński, Decomposition methods, in: Stochastic Programming in: Handbooks in Operations Research and Management Science, Elsevier, Amsterdam, 2003, pp. 141-211. (chapter 3).

[20] S. Sen, Zhihong Zhou, Multistage stochastic decomposition: a bridge between stochastic programming and approximate dynamic programming, SIAM Journal on Optimization (in press).

[21] A. Shapiro, Inference of statistical bounds for multistage stochastic programming problems, Mathematical Methods of Operations Research 58 (2003) 57-68.

[22] A. Shapiro, Analysis of stochastic dual dynamic programming method, European Journal of Operational Research 209 (2011) 63-72. 